\title{
PVRIG is a novel natural killer cell immune checkpoint receptor in acute myeloid leukemia
}

\section{Jessica Li, ${ }^{1,2}$ Sarah Whelan, ${ }^{3}$ Maya F. Kotturi, ${ }^{3}$ Deborah Meyran, $, 1,2,4$ Criselle D'Souza, ${ }^{1,2}$ Kyle Hansen, ${ }^{3}$ Spencer Liang, ${ }^{3}$ John Hunter, ${ }^{3}$ Joseph A. Trapani ${ }^{1,2 \#}$ and Paul J. Neeson ${ }^{1,2 \#}$}

${ }^{1}$ Cancer Immunology Program, Peter MacCallum Cancer Center Melbourne, Victoria, Australia; ${ }^{12}$ Sir Peter MacCallum Department of Oncology, The University of Melbourne, Melbourne, Victoria, Australia; ${ }^{3}$ Compugen, USA, Inc., South San Francisco, CA, USA and ${ }^{4}$ Université de Paris, INSERM, U976 HIPI Unit, Institut de Recherche Saint-Louis, Paris, France

"JAT and PJN contributed equally as co-senior authors.

\section{ABSTRACT}

T his study explored the novel immune checkpoint poliovirus receptor-related immunoglobulin domain-containing (PVRIG) in acute myeloid leukemia (AML). We showed that AML patient blasts consistently expressed the PVRIG ligand (poliovirus receptor-related 2, PVRL2). Furthermore, PVRIG blockade significantly enhanced naural killer (NK)-cell killing of PVRL2 ${ }^{+}$, poliovirus receptor (PVR) ${ }^{\text {lo }}$ AML cell lines, and significantly increased NK-cell activation and degranulation in the context of patient primary AML blasts. However, in AML patient bone marrow, NK-cell PVRIG expression levels were not increased. In order to understand how PVRIG blockade might potentially be exploited therapeutically, we investigated the biology of PVRIG and revealed that NK-cell activation resulted in reduced PVRIG expression on the cell surface. This occurred whether NK cells were activated by tumor cell recognition, cytokines (interleukin 2 [IL-2] and IL-12) or activating receptor stimulation (CD16 and NKp46). PVRIG was present at higher levels in the cytoplasm than on the cell surface, particularly on CD56 ${ }^{\text {right }} \mathrm{NK}$ cells, which further increased cytoplasmic PVRIG levels following IL-2 and IL-12 activation. PVRIG was continually transported to the cell surface via the endoplasmic reticulum and Golgi in both unstimulated and activated NK cells. Taken together, our findings suggest that anti-PVRIG blocking antibody functions by binding to surface-bound PVRIG, which undergoes rapid turnover in both unstimulated and activated NK cells. We conclude that the PVRIG-PVRL2 immune checkpoint axis can feasibly be targeted with PVRIG blocking antibody for NK-mediated immunotherapy of PVRL2 ${ }^{+}$AML.

\section{Introduction}

Poliovirus receptor-related immunoglobulin domain-containing (PVRIG) has recently been identified as an immune checkpoint molecule with potential for therapeutic development. ${ }^{1}$ In humans, PVRIG is expressed on T cells (predominantly $\mathrm{CD} 8^{+} \mathrm{T}$ cells) and natural killer (NK) cells, but not on B cells, monocytes or neutrophils. ${ }^{1}$ PVRIG binds to a single ligand, poliovirus receptor-related 2 (PVRL2, also known as CD112 or Nectin-2), and exerts an inhibitory effect on cytotoxic lymphocyte activity, likely via an ITIM-like motif in its intracellular domain. ${ }^{1-3}$ PVRL2 is an adhesion molecule involved in the formation of cell-cell junctions, and is overexpressed in various cancers..$^{48}$ PVRL2 is also a ligand of the co-activating receptor DNAX accessory molecule 1 (DNAM-1) (10 $^{9}$ and weakly binds another inhibitory receptor, T- cell immunoreceptor with Ig and ITIM domains (TIGIT) ${ }^{11-13}$ Recently, Whelan et al. demonstrated the inhibitory effect of PVRL2 was predominantly mediated by PVRIG and not TIGIT. ${ }^{3}$ DNAM-1 and TIGIT (but not PVRIG) also bind to a closely related molecule, poliovirus receptor (PVR, also known as CD155 or Necl-5). ${ }^{9,11,12}$ Competition studies have determined that PVR has higher affinity for TIGIT than DNAM-1, and PVRL2 has a higher affinity for PVRIG than DNAM-
Haematologica 2021

Volume 106(12):3115-3124

\section{Correspondence:}

PAUL NEESON

paul.neeson@petermac.org

Received: May 19, 2020.

Accepted: October 12, 2020.

Pre-published: November 5, 2020.

https://doi.org/10.3324/haematol.2020.258574

(C2021 Ferrata Storti Foundation

Material published in Haematologica is covered by copyright. All rights are reserved to the Ferrata Storti Foundation. Use of published material is allowed under the following terms and conditions:

https://creativecommons.org/licenses/by-nc/4.0/legalcode. Copies of published material are allowed for personal or internal use. Sharing published material for non-commercial purposes is subject to the following conditions: https://creativecommons.org/licenses/by-nc/4.0/legalcode, sect. 3. Reproducing and sharing published material for commercial purposes is not allowed without permission in writing from the publisher. 
1 , suggesting that the inhibitory signal is dominant.,11

PVRIG inhibitory function was shown using antiPVRIG blocking antibodies. Xu et al. demonstrated that PVRIG blocking antibodies significantly increased NK-cell cytotoxicity against breast cancer cell lines in vitro, an effect that was further enhanced when used in combination with TIGIT blocking antibodies. ${ }^{2}$ An independent group using a different anti-PVRIG antibody similarly showed that PVRIG blockade enhanced T-cell cytotoxicity against melanoma and pancreatic cancer cell lines, which was also augmented by combination with TIGIT blockade. ${ }^{3}$ Notably, Whelan et al. demonstrated that $T$ cells isolated from patient tumors and activated via CD3 increased interferon $\gamma$ production in response to combination PVRIG/TIGIT blockade. ${ }^{3}$ PVRIG blockade also reduced tumour burden in a mouse model when combined with anti-PDL1. ${ }^{14}$ On the basis of these data, a human IgG4 anti-PVRIG blocking antibody is currently undergoing phase I clinical trials in patients with advanced solid tumors. ${ }^{15}$

As PVRIG is present on both T cells and NK cells, blocking PVRIG provides the opportunity to augment both major cytotoxic effector cell types. Although many studies focused on the capacity for immune checkpoint blockade to enhance T-cell responses, the contribution of NK cells should not be overlooked. For instance, tumors often downregulate human leukocyte antigen (HLA) class I to evade CD8 ${ }^{+}$T-cell recognition. ${ }^{16}$ However, this simultaneously removes the ligand for killer cell immunoglobulinlike receptors (KIRs) on NK cells, rendering tumors more sensitive to NK cell-mediated killing. ${ }^{17}$ Reducing the inhibitory signal from KIR has also been shown to be effective in controlling acute myeloid leukemia (AML). AML is an aggressive disease in which myeloid progenitor cells proliferate uncontrollably, and which is frequently treated with allogeneic hematopoietic stem cell transplant (alloHSCT) when patients relapse after front-line chemotherapy. In a seminal study of allo-HSCT patients, mismatches between KIR on donor NK cells and recipient HLA was a key predictor of survival. ${ }^{18,19}$ Recipients lacking HLA ligands for one or more of the KIR expressed by the donor experienced graft-versus-host NK alloreactivity, which was significantly associated with a lower relapse rate. ${ }^{19,20}$

Given the pivotal role of NK cells in AML, strategies to enhance NK-cell activity could provide significant benefit for patients with AML, who have a 5-year survival rate of less than $30 \%$ with current treatments. ${ }^{21}$ This study aimed to determine whether PVRIG blockade could be used to enhance NK-cell responses against AML. Using healthy donor and AML patient blood or bone marrow samples, we evaluated the expression of PVRIG and PVRL2 on NK cells and AML blasts respectively. We also investigated whether PVRIG blockade could enhance NK-cell-mediated killing of AML blasts, and the kinetics of PVRIG surface expression to reveal when the target is expressed following AML target cell activation of NK cells.

\section{Methods}

\section{Reagents and cell lines}

Anti-PVRIG and anti-TIGIT blocking antibodies were provided by Compugen, USA, Inc. Anti-DNAM-1 (11A8), anti-CD16 (3G8), anti-NKp46 (9E2), anti-2B4 (eBioC1.7) and anti-NKG2D (1D11) purified antibodies were purchased from Biolegend.
Recombinant human IL-2, IL-12, IL-15 and IL-18 were purchased from Peprotech. Monensin (GolgiStop, BD Biosciences) and brefeldin A (eBioscience) were both used at 1:1,000. Antibodies used for flow cytometry staining are listed in the Online Supplementary Table S1. SKBR3, KG1a, K562, ML-2, THP-1 and Kasumi-1 cell lines were maintained in RPMI 1640 (Gibco) supplemented with Glutamax, penicillin, streptomycin and 10\% (or $20 \%$ for Kasumi-1) fetal calf serum (FCS). AML-193 cell line was maintained in Iscove's Modified Dulbecco's Media supplemented with $5 \%$ FCS, $5 \mu \mathrm{g} / \mathrm{mL}$ transferrin, $5 \mu \mathrm{g} / \mathrm{mL}$ insulin and $2 \mathrm{ng} / \mathrm{mL}$ granulocyte-macrophage colony-stimulating factor. All cell lines tested negative for mycoplasma.

\section{Acute myeloid leukemia patient and healthy donor bone marrow samples}

All patient and healthy donor samples were obtained under ethics approval from the Peter MacCallum Cancer Center human ethics committee (HREC approval numbers 01/14 and 10-61). Cryopreserved AML patient diagnostic bone marrow samples were obtained from the Cancer Collaborative Biobank (Metro South Health, Queensland, Australia). Patient clinical characteristics are summarised in the Online Supplementary Table S2. Healthy donor bone marrow samples were obtained from Royal Melbourne Hospital (Melbourne, Australia) or purchased from AllCells (Alameda, California). All bone marrow samples were used for flow cytometry staining immediately after thawing.

\section{Healthy donor peripheral blood mononuclear cells and natural killer cells}

Peripheral blood mononuclear cells (PBMC) were isolated from healthy donor buffy coats (Australian Red Cross Blood Service) by density gradient (Ficoll-Paque, GE Healthcare Life Science) and cryopreserved. One day prior to experiments, PBMC were thawed and treated with DNase I (Merck) for 15 minutes at $37^{\circ} \mathrm{C}$. Where required, NK cells were isolated by negative selection using a human NK Cell Isolation Kit (Miltenyi Biotec) according to the manufacturer's instructions (except antibodies and beads were used at half the recommended concentration). The purity of NK cells as determined by flow cytometry was $>95 \%$. Bulk $\mathrm{PBMC}$ or isolated NK cells were incubated in media containing 25 $\mathrm{U} / \mathrm{mL}$ IL-2 overnight at $37^{\circ} \mathrm{C}$ before use in assays.

\section{Natural killer cell stimulation}

Isolated $\mathrm{NK}$ cells were incubated at $37^{\circ} \mathrm{C}$ alone, with the specified combination of cytokines, with target cells at a 1:1 ratio, or in wells precoated (overnight $4^{\circ} \mathrm{C}$ ) with agonistic antibodies against CD16, NKp46, 2B4 or NKG2D. After $24 \mathrm{hr}$, cells were washed and stained with LIVE/DEAD Fixable Yellow (ThermoFisher) followed by antibodies against CD56, CD16, CD69, PVRIG, TIGIT and DNAM-1. For analysis of short term kinetics, cells were incubated at $37^{\circ} \mathrm{C}$ with the indicated stimuli, and at the specified time points were transferred to $4^{\circ} \mathrm{C}$. Cells for the 0 time point were kept at $4^{\circ} \mathrm{C}$. Upon completion of all time points, cells were stained with LIVE/DEAD Fixable Yellow followed by antibodies against CD56, CD16, CD69 and PVRIG.

\section{Other methods}

Details of experimental procedures (flow cytometry, Chromium release assay, degranulation assay) are provided in the Online Supplementary Methods.

\section{Data analysis}

Flow cytometry data were analysed using FlowJo software (BD), and statistical analysis was performed in Prism (GraphPad). Figure 4G was created with BioRender.com. 


\section{Results}

PVRIG blockade enhances natural killer cell killing of PVRL2 ${ }^{\text {hi }}$ PVR ${ }^{10}$ acute myeloid leukemia cells

In order to assess whether PVRIG blockade could enhance NK-cell responses against AML, we utilized the AML cell line KG1a. When co-cultured with healthy donor PBMC and PVRIG blocking antibody, a significant increase in KG1a cell death was observed compared to the untreated control (Figure 1A). In contrast, TIGIT blocking antibody did not significantly enhance KG1a target cell lysis, and KG1a lysis in the presence of combined antiPVRIG and anti-TIGIT was comparable to PVRIG blockade alone (Figure 1A). In order to compare across donors with variable baseline killing, we calculated the NK:target ratio required for $10 \%$ KG1a lysis for each donor. PVRIG
A
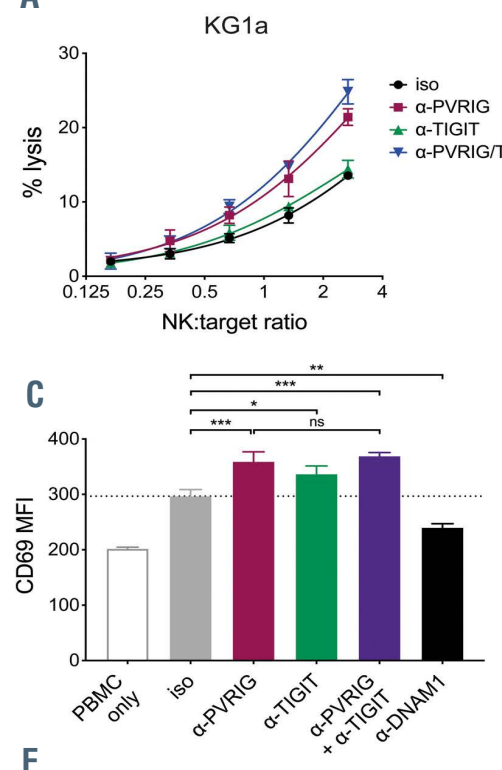

$\mathrm{F}$

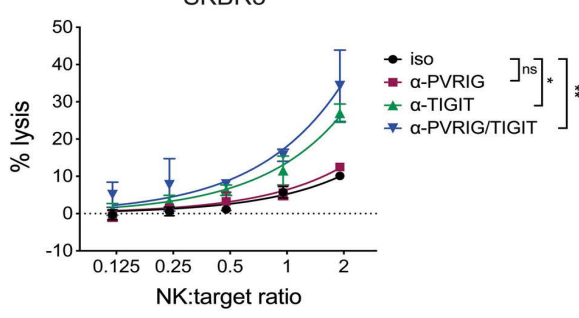

H

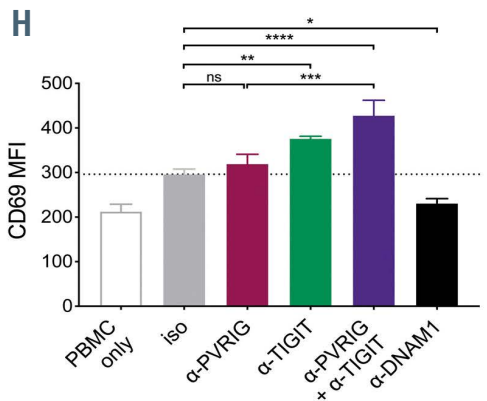

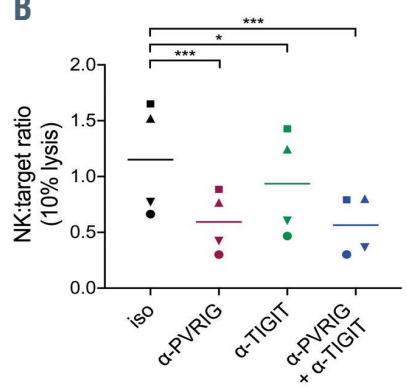

D
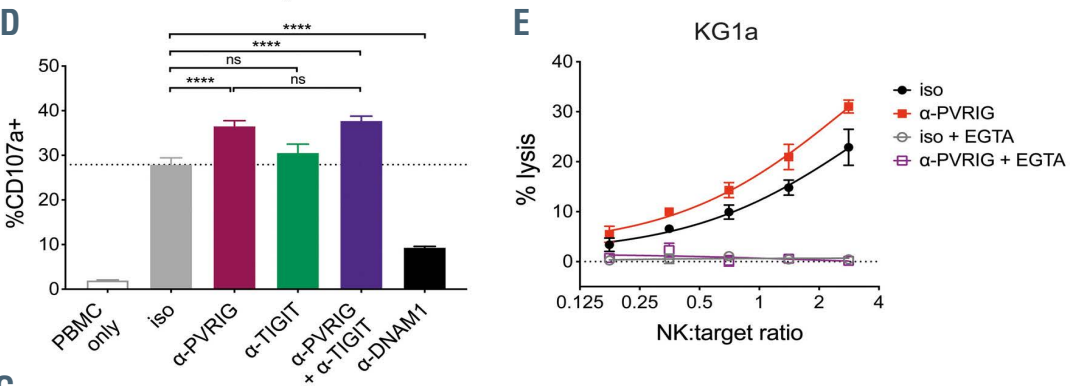

G

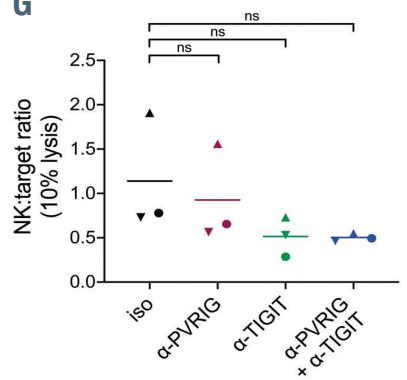

J
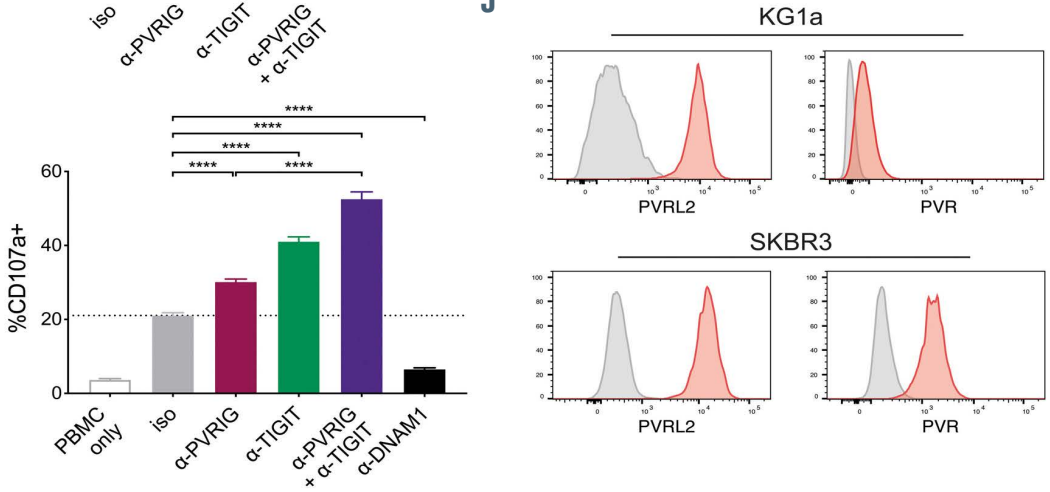

Figure 1. Blockade of PVRIG enhanced natural killer cell killing of tumor cell lines. (A) Percentage lysis of KG1a cells after 4-hour co-culture with peripheral blood mononuclear cells (PBMC) in the presence of anti-PVRIG, anti-TIGIT, anti-PVRIG + anti-TIGIT, or isotype antibodies, measured by ${ }^{51} \mathrm{Cr}$ release assay. Representative data (mean \pm standard deviation [SD] of triplicates) of four experiments shown. (B) Natural killer (NK):target ratio required to achieve $10 \%$ lysis, determined by nonlinear regression of curves plotted as in (A). Each symbol represents an individual donor, $n=4$. NK-cell expression of (C) CD69 and (D) CD107a after 4-hour co-culture of PBMC with KG1a (8:1 E:T ratio) in the presence of the indicated blocking antibodies or isotype control antibody. Representative data (mean \pm SD of triplicates) of two experiments is shown. (E) Percentage lysis of KG1a cells after 4-hour co-culture with PBMC in the presence of anti-PVRIG or isotype antibodies, with or without $4 \mathrm{mM}$ EGTA. Representative data (mean \pm SD of triplicates) of two experiments is shown. (F) Percentage lysis of SKBR3 cells after 4-hour co-culture with PBMC in the presence of anti-PVRIG, anti-TIGIT, anti-PVRIG + anti-TIGIT, or isotype antibodies, measured by ${ }^{51} \mathrm{Cr}$ release assay. Representative data (mean \pm SD of triplicates) of three experiments is shown. (G) NK:target ratio required to achieve $10 \%$ lysis, determined by non-linear regression of curves plotted as in (F). Each symbol represents an individual donor $(n=3)$. NK-cell expression of $(\mathrm{H}) \mathrm{CD} 69$ and $(\mathrm{I}) \mathrm{CD} 107$ a after 4-hour co-culture of PBMC with SKBR3 (2:1 E:T ratio) in the presence of the indicated blocking antibodies or isotype control antibody. Representative data (mean \pm SD of triplicates) of two experiments is shown. (J) Expression of PVRL2 and PVR (red histograms) on SKBR3 and KG1a cells compared with isotype control stain (grey histograms). NK:target ratios in (A, E and F) were calculated using \% of NK cells in PBMC determined by flow cytometry. Significance was determined by repeated measures one-way ANOVA (A, B, $F$ and $G$ ) or one-way ANOVA (C, D, $\mathrm{H}$ and $I$ ) with Holm-Sidak's multiple comparisons test, not significant (ns) $P>0.05, * P<0.05, * * P<0.01, * * * P<0.001, * * * * P<0.0001$. PVRIG: poliovirus receptor-related immunoglobulin domain-containing; TIGIT: T- cell immunoreceptor with Ig and ITIM domains; MFI: mean fluorescence intensity. 
blockade significantly decreased the NK:target ratio required to reach $10 \%$ KG1a lysis, while TIGIT blockade had only a minor effect (Figure 1B). PVRIG blockade also significantly increased NK-cell activation and degranulation, as measured by CD69 and CD107a staining respectively (Figure 1C and D). TIGIT blockade had minimal effect on both NK-cell activation and degranulation and combined PVRIG and TIGIT blockade showed no benefit over PVRIG blockade alone (Figure $1 \mathrm{C}$ and D). The activating receptor DNAM-1 was important for recognition of targets, as blocking DNAM-1 significantly inhibited NKcell activation and degranulation (Figure 1C ,D, H and I). KG1a target cell death was perforin-dependent, as it was completely blocked when free calcium was complexed with EGTA (Figure 1E).

PVRIG blockade was clearly more effective than TIGIT blockade for enhancing NK-cell responses against KG1a but did not enhance lysis of the breast cancer cell line
SKBR3. Rather, significantly more target cell death was observed with TIGIT blockade, or combined PVRIG and TIGIT blockade (Figure 1F). Pooled data from three donors suggested TIGIT blockade, but not PVRIG blockade, decreased the NK:target ratio required for $10 \%$ lysis, but the difference did not reach statistical significance (Figure 1G). TIGIT blockade significantly enhanced NK-cell activation and degranulation, whereas PVRIG blockade had minimal effect on activation and a much smaller effect on degranulation (Figure $1 \mathrm{H}$ and I). Combined PVRIG and TIGIT blockade enhanced NK-cell activation and degranulation cytotoxicity even further, suggesting that PVRIG blockade can have an additive effect to TIGIT blockade (Figure $1 \mathrm{H}$ and $\mathrm{I}$ ).

NK cells from all healthy donors tested expressed both PVRIG and TIGIT (Online Supplementary Figure S1). Although the levels of expression (particularly of TIGIT) varied, all donors were consistently more responsive to
A

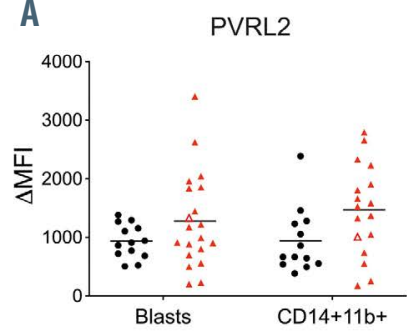

D

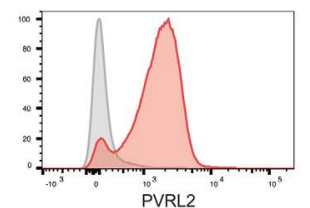

G

HD vs. AML 1

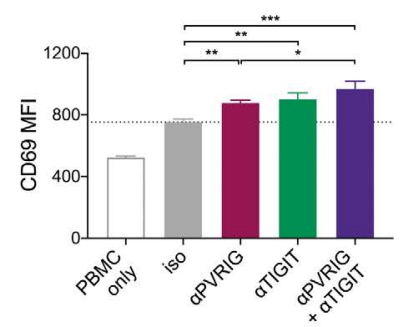

$J$

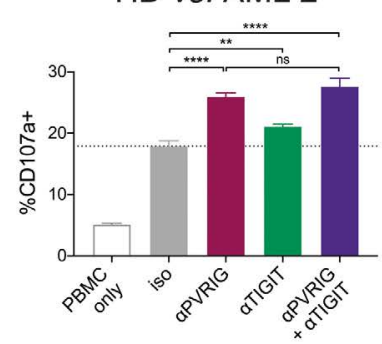

B

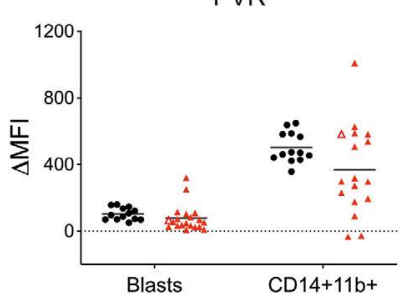

E

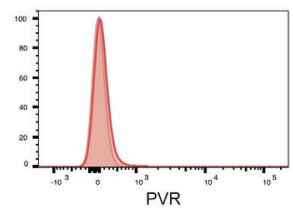

HD vs. AML 1

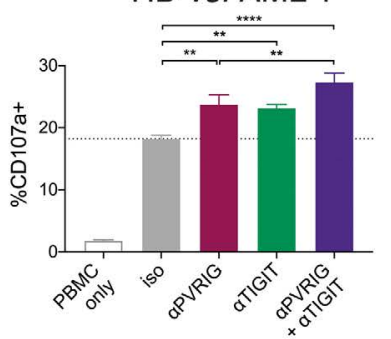

K

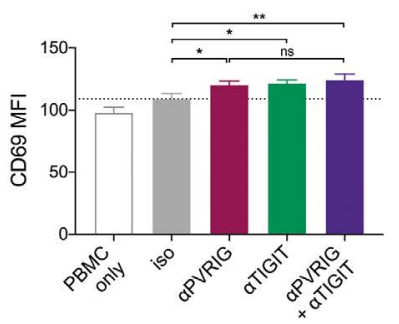

C PVRIG

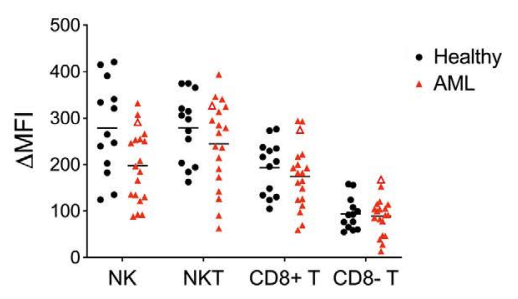

F

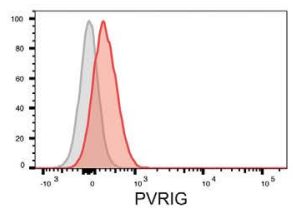

HD vs. AML 2

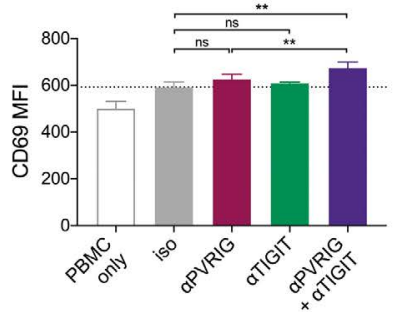

$\mathrm{L}$

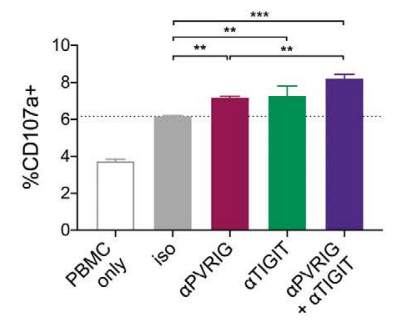

Figure 2. PVRIG and its ligand PVRL2 are expressed in acute myeloid leukemia patient bone marrow. Expression of (A) poliovirus receptor-related 2 (PVRL2) (B) poliovirus receptor (PVR) or (C) poliovirus receptor-related immunoglobulin domain-containing (PVRIG) on blasts or immune cell types in the bone marrow of acute myeloid leukemia (AML) patients $(n=19-20)$ or healthy donors $(n=13)$. Open triangles mark the patient shown in (D to F). Representative histograms of (D) PVRL2 and (E) PVR on AML blasts, or (F) PVRIG on natural killer (NK) cells in the bone marrow of an AML patient. Overlay histograms of test (red) and isotype control stains (grey) are shown. NK-cell expression of (G, I and K) CD69 and (H, J and L) CD107a after 4-hour co-culture of healthy donor peripheral blood mononuclear cells (PBMC) with AML patient bone marrow (8:1 E:T ratio) in the presence of the indicated blocking antibodies or isotype control antibody (mean \pm standard deviation of triplicates, $\mathrm{n}=3$ patients). Significance was determined by one-way ANOVA with Holm-Sidak's multiple comparisons test, not significant (ns) $P>0.05, * P<0.05, * \star P<0.01$, $* * * P<0.001, * * * * P<0.0001 ; \mathrm{MFI}$ : mean fluorescence intensity. 
PVRIG blockade against KG1a targets, and more responsive to TIGIT blockade against SKBR3 targets. Because TIGIT binds preferentially to PVR, while PVRIG binds exclusively to PVRL2, we explored whether differential expression of the ligands on KG1a and SKBR3 could explain the different NK-cell responses to PVRIG or TIGIT blockade. Indeed, we observed that while both SKBR3 and KG1a cells have high expression of PVRL2, KG1a expressed far less PVR than SKBR3 (Figure 1J). This suggests that tumors expressing high levels of PVRL2 but low levels of PVR are more likely to inhibit immune cells via PVRIG, whereas when both ligands are present, inhibition via TIGIT appears to predominate. This trend was also observed with other AML cell lines (Online Supplementary Figure S2). Both AML-193 and Kasumi-1 cells were PVRL2 ${ }^{+}$VRR $^{\text {lo }}$ (Online Supplementary Figure S2A) and were killed at significantly higher levels in the context of antiPVRIG with PBMC from healthy donors (Online Supplementary Figure S2B and C). By contrast, ML-2 and THP-1 AML cells were PVRL2+PVR ${ }^{+}$(Online Supplementary Figure S2A) and were significantly increased killed in the presence of anti-TIGIT rather than anti-PVRIG (Online Supplementary Figure S2D and E).

\section{Acute myeloid leukemia patient bone marrow contains} PVRL2 $^{\text {hi }}$ PVR $^{\text {I0 }}$ blasts and PVRIG ${ }^{+}$natural killer cells

We next examined the expression of PVRIG, PVR and PVRL2 in AML patient bone marrow. Using multicolour flow cytometry, we distinguished various lymphoid $\left(\mathrm{CD}^{-} \mathrm{CD}^{-} 6^{+} \mathrm{NK}\right.$ cells, $\mathrm{CD}^{+} \mathrm{CD}^{-} 6^{+} \mathrm{NKT}$ cells, $\mathrm{CD}^{+} \mathrm{CD}^{+}$ $\mathrm{T}$ cells, $\mathrm{CD}^{+} \mathrm{CD}^{-} \mathrm{T}$ cells), myeloid $\left(\mathrm{SSC}^{\text {hi }} \mathrm{CD} 14^{+} \mathrm{CD} 11 \mathrm{~b}^{+}\right.$ monocytes) and blast (CD45 $\left.{ }^{\text {lo }} S S C^{\text {int }}\right)$ populations collected at diagnosis (Online Supplementary Figure S3). Primary AML blasts were PVRL2 ${ }^{+} \mathrm{PVR}^{\mathrm{lo}}$, and PVR was expressed at higher levels on $\mathrm{CD} 14^{+} \mathrm{CD} 11 \mathrm{~b}^{+}$monocytes (Figure $2 \mathrm{~A}, \mathrm{~B}$, $\mathrm{D}$ and $\mathrm{E}$ ). In healthy donor bone marrow samples, the CD $45^{\text {lo }}$ SSC ${ }^{\text {int }}$ immature myeloid population (gated as per Online Supplementary Figure S3) were also PVRL2+PVR ${ }^{10}$, suggesting that high PVRL2 expression is a feature of normal myeloblasts (Figure 2A and B). Nonetheless, the PVRL2 ${ }^{\text {hipVR }}{ }^{\text {lo }}$ phenotype of the AML blasts suggested they would be a good target for PVRIG blockade, provided that patient effector cells expressed PVRIG. T cells and NK cells expressed PVRIG in all AML patients tested, with higher expression in NK, NKT and CD8 ${ }^{+} \mathrm{T}$ cells and lower expression in CD8- $\mathrm{T}$ cells (Figure $2 \mathrm{C}$ and $\mathrm{F}$ ). There was no statistical difference in PVRIG expression levels on bone
A

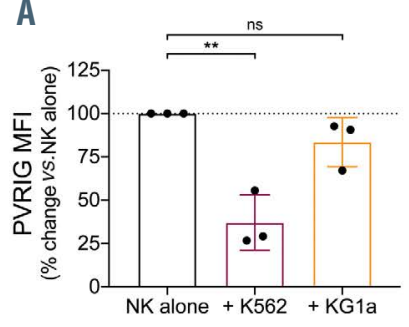

D

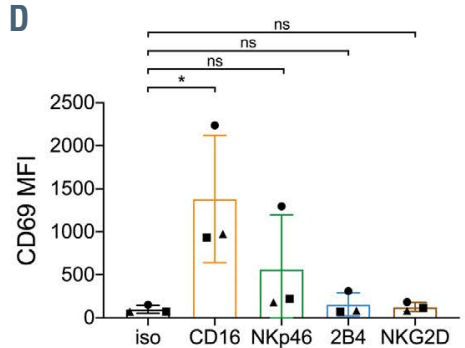

B

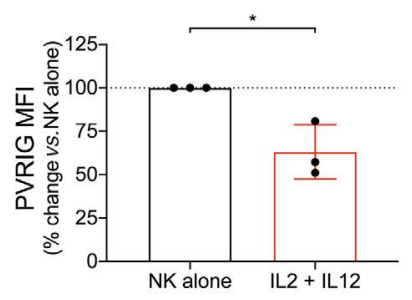

E

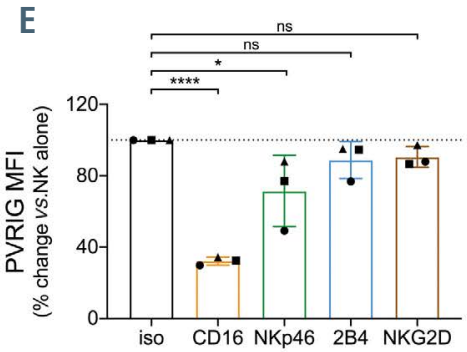

C

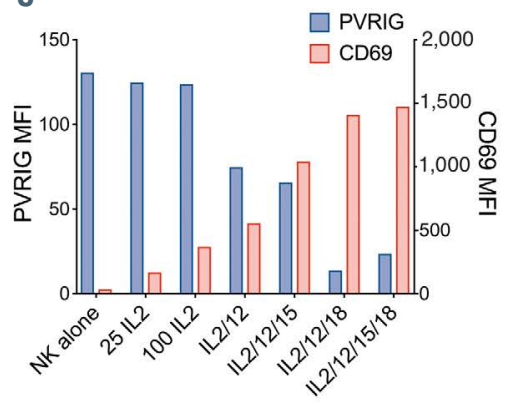

F

NK alone

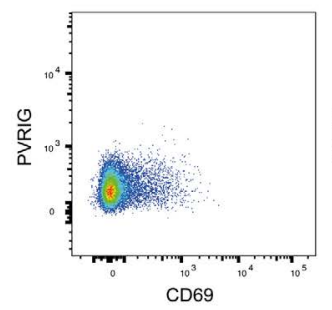

anti-CD16

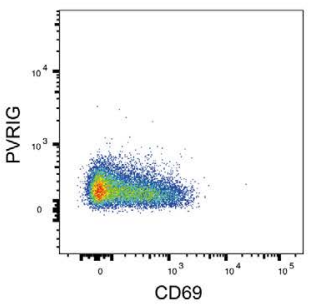

anti-NKp46

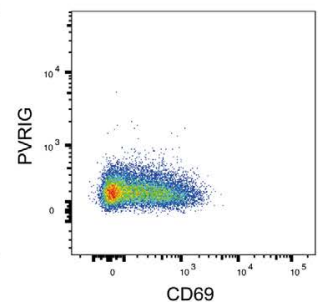

IL2/12

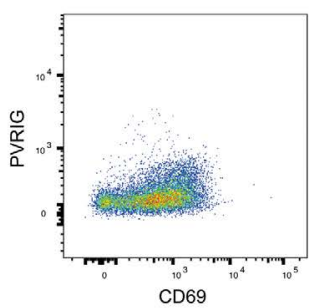

IL2/12/15/18

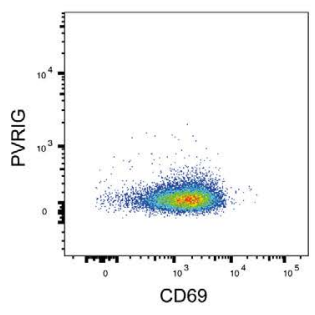

Figure 3. PVRIG expression on natural killer cells is decreased upon activation. (A) Poliovirus receptor-related immunoglobulin domain-containing (PVRIG) expression on isolated natural killer (NK) cells after 24-hour cultured alone, or co-cultured with K562 or KG1a cells (1:1 ratio). Shown is percentage change in PVRIG mean fluorescence intensity (MFI) relative to NK alone, each point represents an individual donor ( $n=3$ ), bars represent mean \pm standard deviation (SD). (B) PVRIG expression on isolated NK cells after 24-hour incubation alone or in the presence of $100 \mathrm{U} / \mathrm{mL}$ interleukin 2 (IL-2) and $10 \mathrm{ng} / \mathrm{mL}$ IL-12. Shown is percentage change in PVRIG MFI relative to NK alone, each point represents an individual donor ( $n=3)$, bars represent mean \pm SD. (C) Expression of PVRIG and CD69 on isolated NK cells after 24-hour incubation with $25 \mathrm{U} / \mathrm{mL}$ IL-2, $100 \mathrm{U} / \mathrm{mL}$ IL-2, or combinations of IL-2 (100 U/mL), IL-12 (10 ng/mL), IL-15 (50 ng/mL) and IL-18 (50 ng/mL), as indicated. Representative data of two independent experiments is shown. Expression of (D) CD69 or (E) PVRIG on isolated NK cells after 24-hour incubation with the indicated plate-bound antibodies. Shown is percentage change in PVRIG MFI relative to isotype. Each donor is represented by a distinct symbol ( $n=3$ ), bars represent mean \pm SD. (F) Expression of PVRIG vs. CD69 on isolated NK cells after 24-hour incubation with indicated stimuli, as in (C) and (E). Significance determined by one-way ANOVA with Holm-Sidak's multiple comparisons test (A, Dand E) or Student's $t$-test (B), not significant (ns) $P>0.05, * P<0.05, * \star P<0.01, * * * P<0.001, * * * \star P<0.0001$. 
marrow immune subsets of AML patients compared with healthy donors (Figure 2C). PVRIG and PVRL2 expression levels varied considerably amongst patients, but this did not correlate with AML subtype or the percentage of bone marrow blasts (Online Supplementary Figure S4).

In order to assess whether PVRIG blockade could enhance NK-cell killing of AML patient blasts, we co-cultured healthy donor PBMC with bone marrow from an AML patient with a high percentage $(>90 \%)$ of PVRL2 ${ }^{\text {hiPVR }}{ }^{\text {lo }}$ AML blasts. In this context, NK cells showed significantly increased CD69 expression and degranulation with PVRIG or TIGIT blockade. In addition, combined PVRIG and TIGIT blockade was associated with significantly higher NK-cell activation and degranulation (Figure $2 \mathrm{G}$ and $\mathrm{H}$ ). Similar results were obtained for a further two AML patients tested (Figure 2I to L). This indicates that PVRIG blockade or combination PVRIG/TIGIT blockade could enhance NK-cell cytotoxicity against PVRL2+ tumor targets in AML patients.

\section{PVRIG expression on natural killer cells is modulated by activation}

In order to understand why PVRIG was not upregulated on AML patient NK cells, we explored the mechanisms regulating NK cell PVRIG expression. In order to do this, we activated healthy donor NK cells for 24 hours via co-culture
A

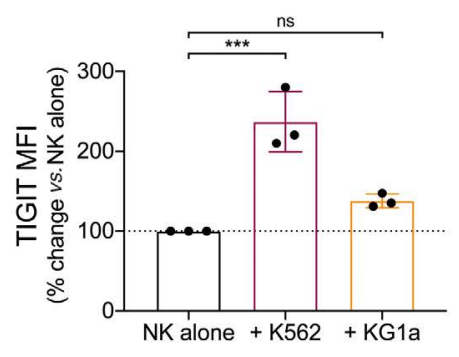

D

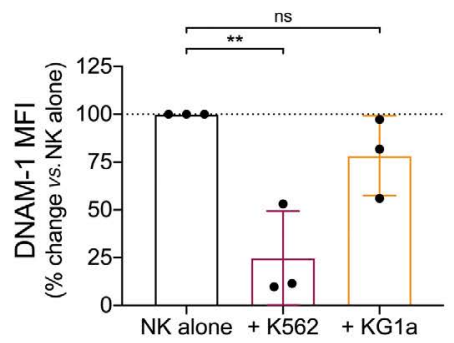

B

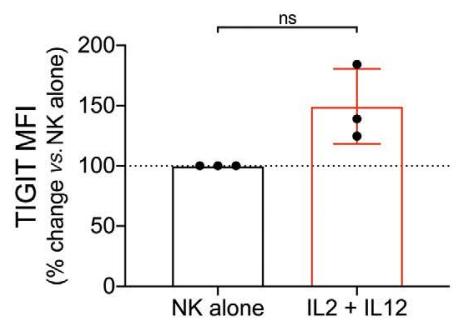

E

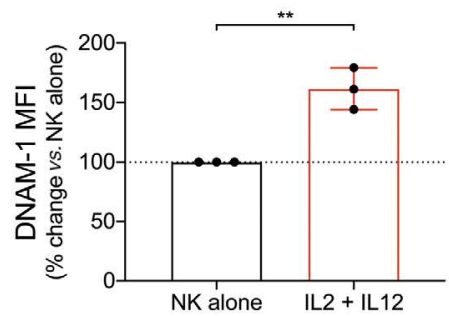

C

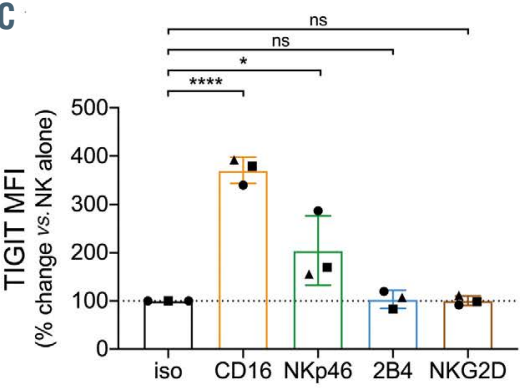

$\mathbf{F}$

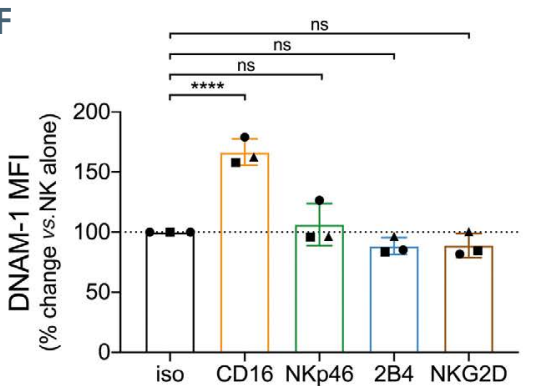

G

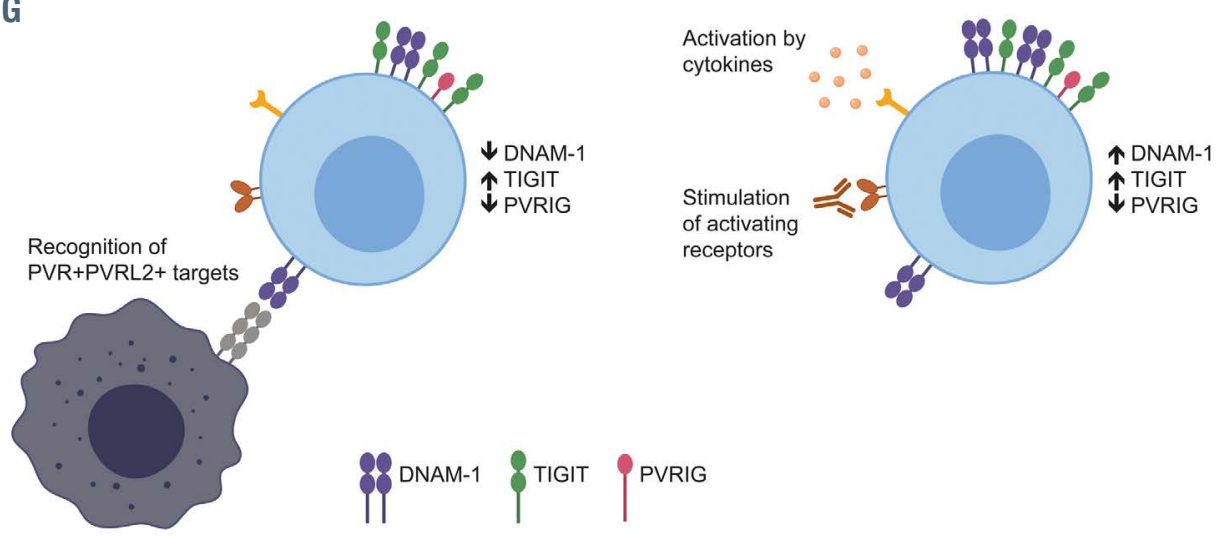

Figure 4. Modulation of DNAM-1 and TIGIT expression on natural killer cells upon activation. (A to C) T-cell immunoreceptor with Ig and ITIM domains (TIGIT) or (D to F) DNAX accessory molecule 1 (DNAM-1) expression on isolated natural killer cells after 24-hour culture with (A and D) K562 or KG1a cells (1:1 ratio); (Band E) $100 \mathrm{U} / \mathrm{mL}$ interleukin 2 (IL-2) and $10 \mathrm{ng} / \mathrm{mL}$ IL-12; or (C and F) with the indicated plate-bound antibodies. Percentage change in mean fluorescence intensity (MFI) relative to natural killer (NK) alone is shown, each point represents an individual donor $(n=3)$, bars represent mean \pm standard deviation (SD). Significance determined by one-way ANOVA with Holm-Sidak's multiple comparisons test (A, C, D and F) or Student's t-test (B and E), not significant (ns) $P>0.05, * P<0.05, * * P<0.01$, $* * * P<0.001, * * * * P<0.0001$. (G) Model of PVRIG-TIGIT-DNAM-1 modulation upon NK-cell activation. Left: upon recognition of tumor cells expressing poliovirus receptor (PVR) and poliovirus receptor-related 2 (PVRL2), NK cells increase TIGIT but lose expression of both poliovirus receptor-related immunoglobulin domain-containing (PVRIG) and DNAM-1. This loss of DNAM-1 may result from a tumor-intrinsic mechanism of immune escape, whereby tumor cells expressing DNAM-1 ligands induce loss of DNAM-1 expression on immune cells. Right: upon activation via cytokines such as IL-2 and IL-12, or by stimulation of activating receptors such as CD16, NK cells decrease expression of PVRIG while increasing expression of TIGIT and DNAM-1. The increased expression of DNAM-1 relative to the decreased expression of PVRIG may serve to push the balance within the cell towards more activating signaling. In this way, PVRIG may act to constitutively dampen NK responsiveness in the steady state and is lost upon activation to lower the activation threshold of the NK cell. 
with tumor targets, NK-cell activating cytokines or via agonistic antibodies to NK-cell activating receptors. NK cells consistently decreased PVRIG expression after interaction with target cells, although to a much greater degree with K562 than KG1a cells (Figure 3A). This may be because K562 lacks human leukocyte antigen (HLA) class I, resulting in greater activation of the NK cells. We next investigated whether activation of NK cells via cytokines would also cause loss of PVRIG. Indeed, NK cells stimulated with IL-2 and IL-12 had significantly decreased PVRIG expression (Figure 3B). Using IL-2 or combinations of IL-2, IL-12, IL-15 and IL-18, NK cells were increasingly activated, as meas- ured by CD69 levels. Interestingly, NK-cell surface PVRIG and CD69 levels were inversely correlated in NK cells undergoing cytokine-mediated activation (Figure 3C). Stimulation of NK cells with plate-bound agonistic antibodies against the activating receptors CD16, NKp46, 2B4 and NKG2D also resulted in differing levels of activation (Figure 3D) and a concomitant decrease in NK-cell PVRIG levels (Figure 3E). When PVRIG and CD69 expression was examined in individual NK cells, there was a trend for more activated NK cells (higher CD69) to express proportionally less PVRIG after anti-CD16 or anti-NKp46 stimulation (Figure 3F). In contrast, following cytokine stimulation NK cells
A

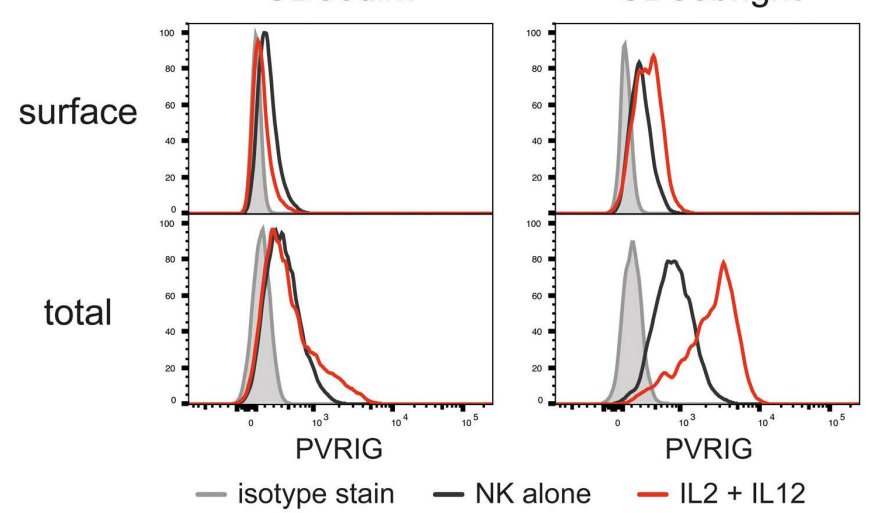

B

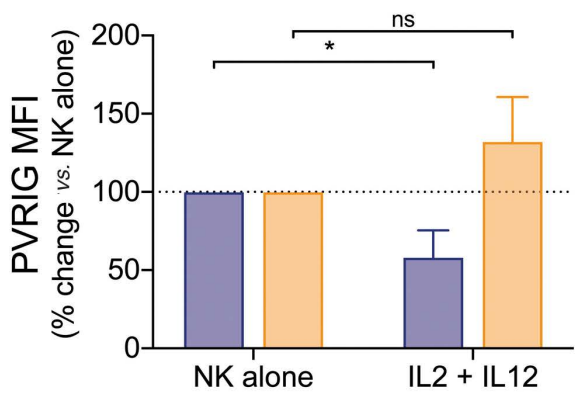

CD56dim

CD56bright

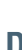

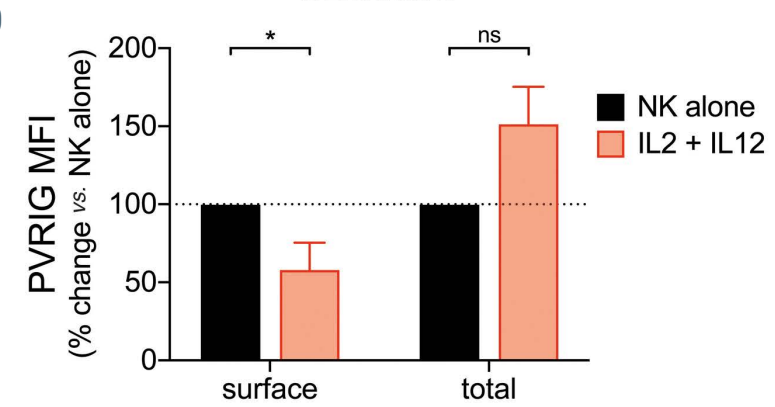

E
C

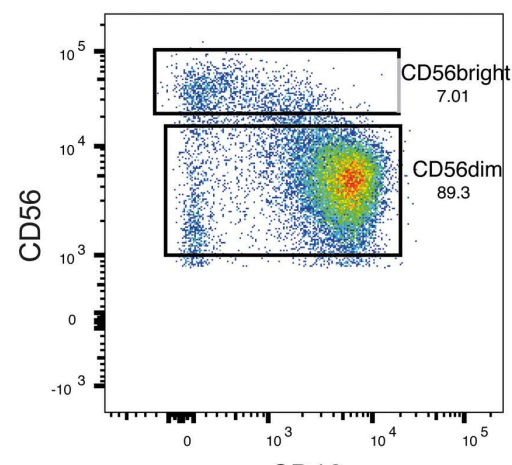

CD16

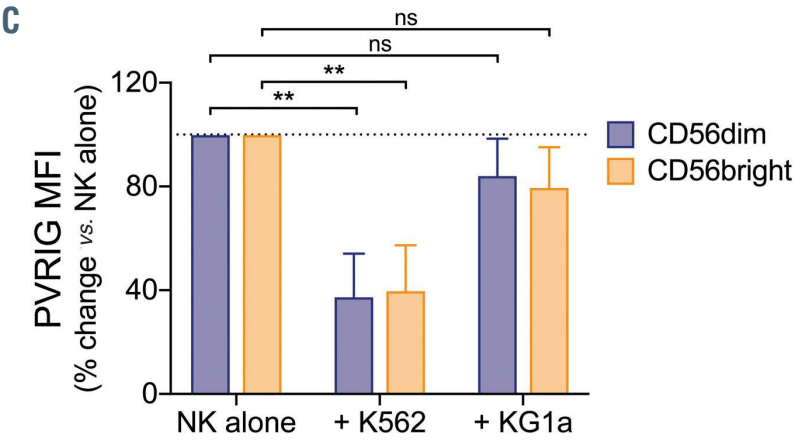
CD56bright

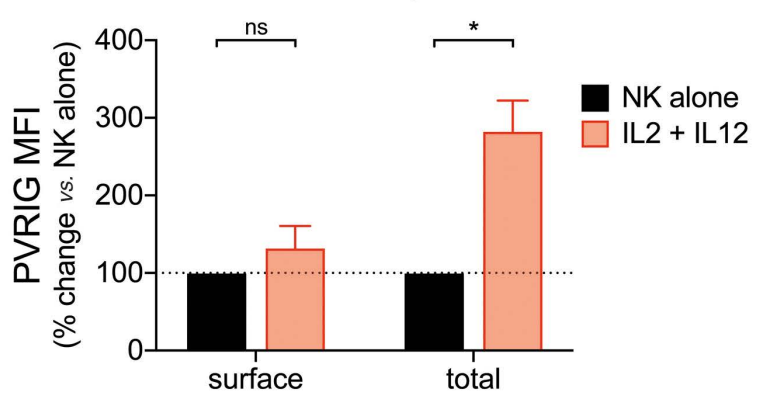

Figure 5. Differential regulation of surface and intracellular PVRIG by CD56 dim and CD56 bright natural killer cells. (A) Poliovirus receptor-related immunoglobulin domain-containing (PVRIG) expression on isolated natural killer (NK) cells (gated on CD56dim or CD56bright subsets) after 24-hour stimulation with 100 U/mL IL-2 and $10 \mathrm{ng} / \mathrm{mL} \mathrm{IL-12,} \mathrm{measured} \mathrm{by} \mathrm{surface} \mathrm{or} \mathrm{total} \mathrm{(intracellular} \mathrm{+} \mathrm{surface)} \mathrm{staining.} \mathrm{Representative} \mathrm{of} \mathrm{two} \mathrm{to} \mathrm{three} \mathrm{experiments.} \mathrm{(B} \mathrm{and} \mathrm{C)} \mathrm{Expression} \mathrm{of} \mathrm{surface}$ PVRIG on isolated NK cells (gated on CD56 ${ }^{\text {dim }}$ or CD56 ${ }^{\text {bright }}$ ) after 24-hour culture with (B) $100 \mathrm{U} / \mathrm{mL}$ interleukin 2 (IL-2 )and $10 \mathrm{ng} / \mathrm{mL}$ IL-12; or (C) with K562 or KG1a cells (1:1 ratio). Shown is percentage change in mean fluorescence intensity (MFI) relative to NK alone, bars represent mean \pm standard deviation (SD) of three experiments. PVRIG expression on NK cells gated on (D) CD56 dim or (E) CD56 bright subsets, measured by surface or total (intracellular + surface) staining after 24hour stimulation with $100 \mathrm{U} / \mathrm{mL} \mathrm{IL}-2$ and $10 \mathrm{ng} / \mathrm{mL} \mathrm{IL-12}$. Shown is the percentage change in PVRIG MFI relative to NK alone, bars represent mean \pm SD of two to three experiments. Significance determined by Student's $t$-test, not significant (ns) $P>0.05, * P<0.05, * * P<0.01$. 
increased CD69 and decreased PVRIG expression more uniformly (Figure 3F).

\section{TIGIT and DNAM-1 expression on natural killer cells is modulated by activation}

TIGIT and DNAM-1 are NK-cell receptors within the same receptor-ligand axis as PVRIG. ${ }^{9-12}$ We investigated whether a similar modulation of TIGIT and DNAM-1 occurred following NK-cell activation. In contrast to PVRIG, TIGIT expression was increased after stimulation of NK cells with target cells, cytokines or agonistic antibodies (Figure 4A to C), whereas changes in DNAM-1 levels were dependent on the stimulus. DNAM-1 was reduced by interaction with target cells (Figure 4D), but increased

A

Surface PVRIG

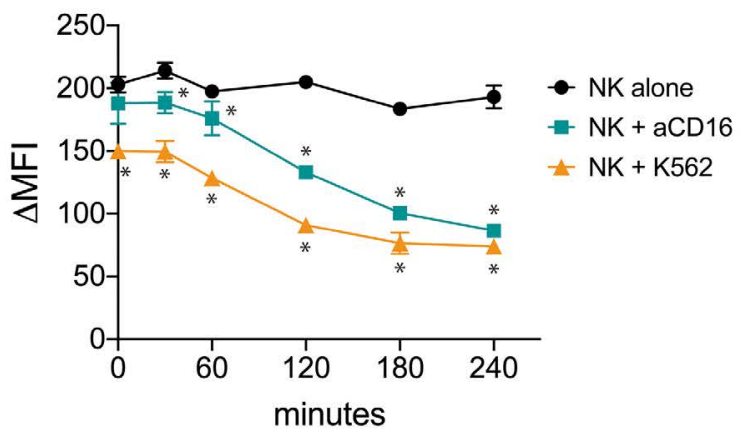

C

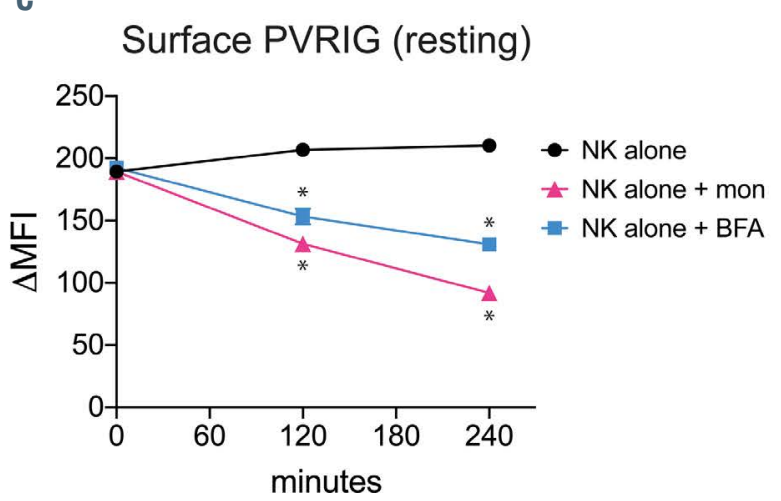

E

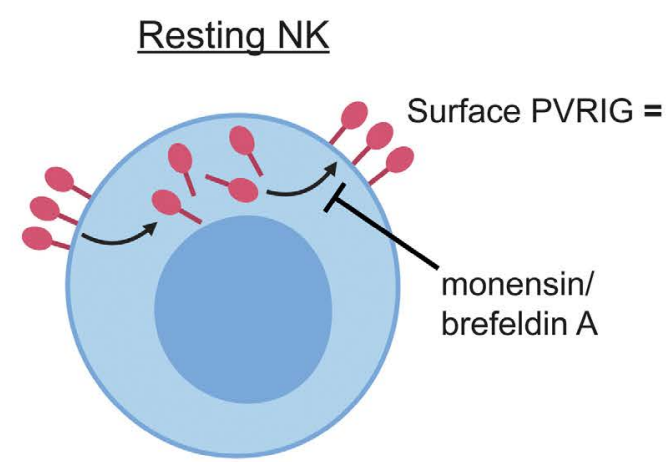

after activation with IL-2 and IL-12 or anti-CD16 (Figure 4E to F). Overall, our results indicated that the expression levels for different NK receptors are regulated differently, depending on the stimulus. PVRIG was consistently decreased and TIGIT increased upon NK-cell activation, regardless of the stimulus, and the magnitude of change was correlated with the level of activation. On the other hand, DNAM-1 expression decreased upon target recognition, but was increased by activation via cytokines or agonistic antibodies to activating receptors (Figure 4G). The loss of DNAM-1 may result from a form of immune evasion, which has previously been described to occur on contact with $\mathrm{PVR}^{+}$tumor cells. ${ }^{22}$ DNAM-1 increase in response to stimulation via cytokines or activation receptors could

B $\%$ CD69+

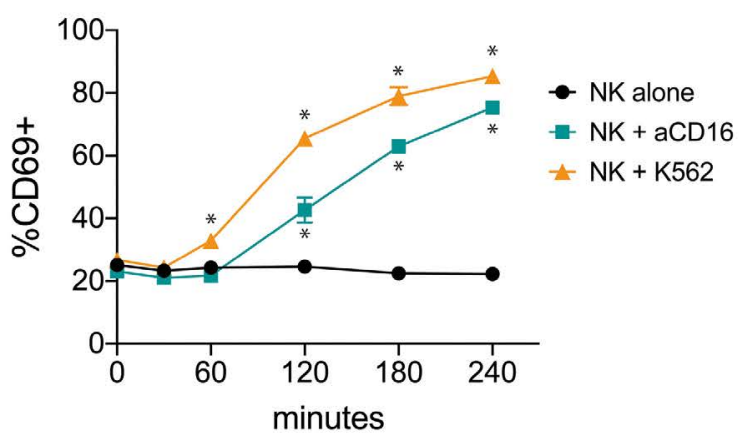

D

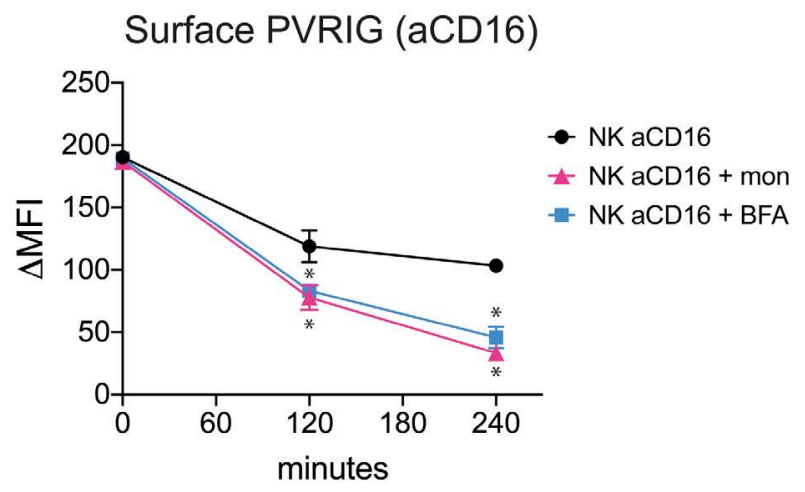

Activated NK

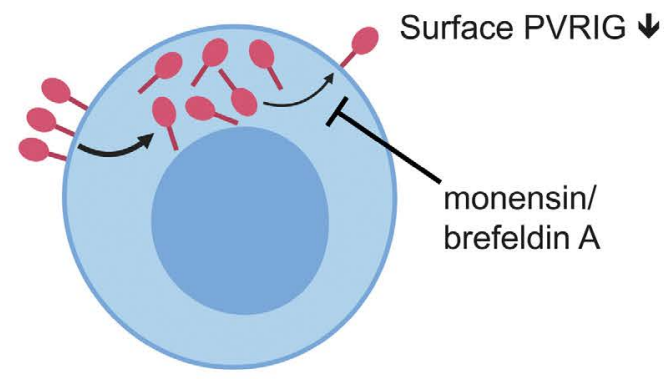

Figure 6. PVRIG is constitutively trafficked to the naural killer cell surface. (A) Poliovirus receptor-related immunoglobulin domain-containing (PVRIG) and (B) CD69 expression on isolated natural killer (NK) cells incubated alone, with $\mathrm{K} 562$ cells $\left(1: 1\right.$ ratio), or with plate-bound anti-CD16 antibody at $37^{\circ} \mathrm{C}$ for the indicated time points. Representative data (mean \pm standard deviation [SD] of duplicates) of two experiments is shown. (C and D) PVRIG expression on isolated NK cells incubated either (C) alone or (D) with plate-bound anti-CD16 antibody at $37^{\circ} \mathrm{C}$ for the indicated time points, in the presence or absence of monensin (mon) or brefeldin $\mathrm{A}(\mathrm{BFA})$. Representative data (mean \pm SD of duplicates) of two experiments is shown. Significance determined by multiple $t$-tests with Holm-Sidak's correction, $\star P<0.05$ compared with NK alone (A and B) or untreated (C and D). 
be a means to increase the net activation signal, which, in conjunction with decreased PVRIG expression, could serve to lower the activation threshold of the NK cell.

\section{Intracellular PVRIG does not decrease upon activation}

We next investigated whether PVRIG levels are regulated differently in the two principal peripheral blood NK-cell subsets, $\mathrm{CD} 56^{\mathrm{dim}} \mathrm{CD} 16^{+}$and $\mathrm{CD} 56^{\text {bright }} \mathrm{CD} 16^{-}$. After IL-2 and IL-12 stimulation, the CD56 $6^{\mathrm{dim}}$ subset showed decreased PVRIG surface expression, as was seen previously for unfractionated NK cells (Figure 5A and B). This was unsurprising given that CD56dim comprise 90-95\% of all circulating NK cells. By contrast, CD56 $6^{\text {bright }} \mathrm{NK}$ cells showed no loss of PVRIG (Figure 5A and B). This was not due to failed activation, as both subsets upregulated CD69 equally (data not shown). Interestingly, this distinct pattern of regulation of PVRIG occurred only with cytokine stimulation (Figure 5B), but not following interaction with target cells, which caused both subsets to downregulate PVRIG (Figure 5C). We next determined whether an intracellular pool of PVRIG exists, or if it is solely expressed on the cell surface. Using permeabilized cells, we detected total (surface plus intracellular) PVRIG levels. For both CD56 $6^{\text {dim }}$ and CD56 ${ }^{\text {bright }}$ NK-cell subsets, total PVRIG staining was far greater than surface staining, indicating that a pool of PVRIG is present in the cytosol (Figure 5A). Interestingly, while CD56dim NK cells lost their surface PVRIG upon activation with IL-2 and IL-12, the total amount of PVRIG was unchanged compared with untreated NK cells (Figure 5D). This suggested that PVRIG lost from the cell surface upon activation was internalized. Alternatively, the total PVRIG level could be maintained despite decreased surface expression by synthesis of new molecules, which then egress to the cell surface. The latter appeared to be the case for CD56bright NK cells, which maintained PVRIG surface expression and increased total PVRIG levels upon activation by IL-2 and IL-12 (Figure 5E).

\section{Natural killer cell surface PVRIG levels are maintained by continuous trafficking to the cell surface}

In order to further explore the mechanisms by which NK cells regulate surface PVRIG levels, we first assessed the kinetics of PVRIG loss from the cell surface under different stimulation conditions using a short term time course. NK cells co-cultured with K562 cells showed loss of PVRIG expression within 1-2 hours, at which time CD69 began to be upregulated (Figure 6A-B). Stimulation of NK cells with anti-CD16 caused a similar level of PVRIG loss and activation, although K562 appeared to be the stronger stimulus at early time points (Figure 6A and B). Within the 4 hours assessed, IL-2 and IL-12 stimulation had no appreciable effect on PVRIG expression, and minimal effect on activation (Online Supplementary Figure S5), indicating that cytokine stimulation influences PVRIG levels more slowly. Next, we used monensin and brefeldin A to determine whether intracellular trafficking via the endoplasmic reticulum (ER) and Golgi network was important for maintaining NK-cell surface PVRIG. Both monensin and brefeldin A inhibit the trafficking of molecules to the cell surface, by disrupting the Golgi apparatus, trans-Golgi network or endosomal network. ${ }^{23-26}$ Untreated NK cells maintained constant PVRIG surface levels over 4 hours. However, the addition of either monensin or brefeldin A resulted in significant loss of surface PVRIG levels (Figure 6C). Interestingly, the presence of monensin or brefeldin A also caused greater loss of PVRIG in NK cells undergoing activation via anti-CD16 stimulation (Figure 6D). These results suggest there is trafficking of PVRIG molecules to the cell surface, via the ER and Golgi, in both untreated and activated NK cells (Figure 6E).

In summary, PVRIG is downregulated on the NK-cell surface following activation by tumor targets, anti-CD16 or cytokines. Furthermore, a pool of PVRIG is present in the NK-cell cytoplasm, and cell surface PVRIG is maintained by trafficking to the surface. Taken together, our findings suggest that anti-PVRIG blocking antibodies enhanced NK-cell killing of AML target cells by blocking PVRIG present on the NK-cell surface. This resulted in decreased PVRL2-PVRIG mediated inhibition, and a decreased threshold for NK-cell activation and increased AML blast killing.

\section{Discussion}

Enhancing the activity of NK cells following HSCT may be beneficial for AML patients. NK cells are the first lymphoid cells to be reconstituted after HSCT, reaching normal levels within 1 month after transplant, much earlier than $\mathrm{T}$ cells. ${ }^{27,28}$ However, their capacity to kill residual leukemic blasts can be limited by the interaction of NKinhibitory receptors with ligands in the tumor microenvironment. ${ }^{27,29,30}$ Thus, blocking inhibitory receptors such as PVRIG could potentially be useful after HSCT to enhance NK-cell activity to delay or prevent relapse.

In this study, we showed PVRIG blockade enhanced human NK-cell activity against PVRL2hiPVRlo AML target cells. AML blasts in patient bone marrow were PVRL2 ${ }^{\text {hiPVR }}{ }^{\text {lo }}$, suggesting PVRIG blockade may increase NK-mediated killing of AML blasts. The AML blast PVRL2 ${ }^{\text {hipVR }}{ }^{\text {lo }}$ phenotype is consistent with previous studies in AML patients. ${ }^{31}$ Our study is the first to report NK cell PVRIG expression in AML patient bone marrow. NK-cell PVRIG expression was not upregulated in AML patients. Our subsequent analysis suggested PVRIG upregulation is not required for PVRIG blockade to be effective. Even though interaction with AML cells caused loss of PVRIG from the NK-cell surface, PVRIG molecules trafficked via the ER-Golgi network and were then expressed on the cell surface. This suggests that, over time, a far greater amount of PVRIG is available on the cell surface to be blocked by anti-PVRIG antibodies than is detected at a single time point.

In contrast to PVRIG, other NK-cell immune checkpoint receptors, such as TIGIT, are upregulated with activation.,11 Despite sharing the same ligand as TIGIT, the modulation of PVRIG does not follow this model, suggesting it could have a distinct biological function. It is possible that PVRIG acts as a regulator to keep NK cells in check in the steady state. The downregulation of PVRIG in response to cytokines or target recognition would then allow greater activation of NK cells in response to inflammatory stimuli. Our data showed NK cell PVRIG was present at higher levels in the cytoplasm than on the cell surface; this intracellular PVRIG was not decreased by activation. This cytoplasmic pool of PVRIG could represent either newly synthesized or recycled protein. A recent study by Whelan et al. ${ }^{3}$ examined PVRIG expression on isolated human $\mathrm{T}$ cells, and observed a similar trend for loss of PVRIG expression immediately after acti- 
vation. However, sustained activation of $\mathrm{T}$ cells with antigen, IL-2 and IL-7 resulted in increased PVRIG expression by day 11 .

This study reveals unique aspects of PVRIG biology that should be considered when determining potential indications for its therapeutic use. Our results suggest that PVRIG blockade may still have a therapeutic effect, provided the tumor cells express the PVRL $2^{\text {hip }}$ SVR ${ }^{\text {lo }}$ phenotype. Furthermore, when AML blasts express both PVRL2 and PVR, combination PVRIG and TIGIT blockade may also induce an effective NK-cell mediated anti-tumor response. These findings also have broader implications for the study of other checkpoint receptors. As more novel receptors are identified as potential targets, they should not be assumed to have the same biology as previously established immune checkpoints, and their potential effi- cacy should not necessarily be measured by the same parameters.

\section{Disclosures}

No conflicts of interest to disclose.

\section{Contributions}

$J L, D M$ and $C D$ performed research, analyzed data and wrote the manuscript; $S W, M K, K H, D M, K H, S L$ and JH provided academic input, shared key reagents, and wrote the manuscript; JAT and PJN supervised the project and wrote the manuscript.

\section{Funding}

Research funding for this project was provided by Compugen Inc. In the prior 36 months, $P N$ also received research funding from BMS, Roche Genentech and Allergan.

\section{References}

1.Zhu Y, Paniccia A, Schulick AC, et al. Identification of CD112R as a novel checkpoint for human $\mathrm{T}$ cells. J Exp Med. 2016;213(2):167-176.

2. Xu F, Sunderland A, Zhou Y, Schulick RD, Edil BH, Zhu Y. Blockade of CD112R and TIGIT signaling sensitizes human natural killer cell functions. Cancer Immunol Immunother. 2017:66(10):1367-1375.

3. Whelan S, Ophir E, Kotturi MF, et al. PVRIG and PVRL2 Are induced in cancer and Inhibit CD8(+) T-cell function. Cancer Immunol Res. 2019;7(2):257-268.

4. Li M, Qiao D, Pu J, Wang W, Zhu W, Liu H. Elevated Nectin-2 expression is involved in esophageal squamous cell carcinoma by promoting cell migration and invasion. Oncol Lett. 2018:15(4):4731-4736.

5. Oshima T, Sato S, Kato J, et al. Nectin-2 is a potential target for antibody therapy of breast and ovarian cancers. Mol Cancer. 2013;12:60.

6. Miao X, Yang ZL, Xiong L, et al. Nectin-2 and DDX3 are biomarkers for metastasis and poor prognosis of squamous cell/adenosquamous carcinomas and adenocarcinoma of gallbladder. Int J Clin Exp Pathol. 2013;6(2):179-190.

7. Liang S, Yang Z, Li D, et al. The clinical and pathological significance of Nectin-2 and DDX3 expression in pancreatic ductal adenocarcinomas. Dis Markers. 2015; 2015:379568.

8. Bekes I, Lob S, Holzheu I, et al. Nectin-2 in ovarian cancer: how is it expressed and what might be its functional role? Cancer Sci. 2019;110(6):1872-1882

9. Bottino C, Castriconi R, Pende D, et al. Identification of PVR (CD155) and Nectin-2 (CD112) as cell surface ligands for the human DNAM-1 (CD226) activating molecule. J Exp Med. 2003;198(4):557-567.

10. Tahara-Hanaoka S, Shibuya K, Onoda Y, et al. Functional characterization of DNAM-1 (CD226) interaction with its ligands PVR (CD155) and nectin-2 (PRR-2/CD112). Int Immunol. 2004;16(4):533-538.

11. Yu X, Harden K, Gonzalez LC, et al. The surface protein TIGIT suppresses $T$ cell activa- tion by promoting the generation of mature immunoregulatory dendritic cells. Nat Immunol. 2009;10(1):48-57.

12. Stanietsky N, Simic H, Arapovic J, et al. The interaction of TIGIT with PVR and PVRL2 inhibits human NK cell cytotoxicity. Proc Natl Acad Sci U S A. 2009; 106(42):1785817863.

13. Deuss FA, Gully BS, Rossjohn J, Berry R. Recognition of nectin- 2 by the natural killer cell receptor $\mathrm{T}$ cell immunoglobulin and ITIM domain (TIGIT). J Biol Chem. 2017;292(27):11413-11422.

14. Murter B, Pan X, Ophir E, et al. Mouse PVRIG Has CD8(+) T cell-specific coinhibitory functions and dampens antitumor immunity. Cancer Immunol Res. 2019;7(2): 244-256.

15. NCT03667716: COM701 in subjects with advanced solid tumors. [cited 22 Jan 2020]; Available from: https:// ClinicalTrials.gov/ show/NCT03667716

16. Aptsiauri N, Ruiz-Cabello F, Garrido F. The transition from HLA-I positive to HLA-I negative primary tumors: the road to escape from T-cell responses. Curr Opin Immunol. 2018;51:123-132.

17. Guillerey C, Smyth MJ. Cancer Immunosurveillance by natural killer cells and other innate lymphoid cells. In: Zitvogel L, Kroemer G, eds. Oncoimmunology: A Practical Guide for Cancer Immunotherapy. Cham: Springer International Publishing, 2018:163-180.

18. Ruggeri L, Capanni M, Casucci M, et al. Role of natural killer cell alloreactivity in HLAmismatched hematopoietic stem cell transplantation. Blood. 1999;94(1):333-339.

19. Ruggeri L, Capanni M, Urbani E, et al. Effectiveness of donor natural killer cell alloreactivity in mismatched hematopoietic transplants. Science. 2002;295(5562):20972100 .

20. Ruggeri L, Mancusi A, Capanni M, et al. Donor natural killer cell allorecognition of missing self in haploidentical hematopoietic transplantation for acute myeloid leukemia: challenging its predictive value. Blood. 2007;110(1):433-440.

21. Howlader N, Noone A, Krapcho M, et al. SEER Cancer Statistics Review, 1975-2016, National Cancer Institute. Bethesda, MD.
Available from https://seer.cancer.gov/ csr/1975_2016/, based on November 2018 SEER data submission, posted to the SEER web site, April 2019.

22. Carlsten M, Norell H, Bryceson YT, et al. Primary human tumor cells expressing CD155 impair tumor targeting by downregulating DNAM-1 on NK cells. J Immunol. 2009:183(8):4921-4930.

23. Mollenhauer HH, Morre DJ, Rowe LD. Alteration of intracellular traffic by monensin; mechanism, specificity and relationship to toxicity. Biochim Biophys Acta. 1990;1031(2):225-246.

24. Tartakoff AM. Perturbation of vesicular traffic with the carboxylic ionophore monensin. Cell. 1983;32(4):1026-1028.

25. Fujiwara T, Oda K, Yokota S, Takatsuki A, Ikehara Y. Brefeldin A causes disassembly of the Golgi complex and accumulation of secretory proteins in the endoplasmic reticulum. J Biol Chem. 1988;263(34):1854518552.

26. Klausner RD, Donaldson JG, LippincottSchwartz J. Brefeldin A: insights into the control of membrane traffic and organelle structure. J Cell Biol. 1992:116(5):1071-1080.

27. Nguyen S, Dhedin N, Vernant JP, et al. NKcell reconstitution after haploidentical hematopoietic stem-cell transplantations: immaturity of NK cells and inhibitory effect of NKG2A override GvL effect. Blood. 2005;105(10):4135-4142.

28. Mancusi A, Ruggeri L, Velardi A. Haploidentical hematopoietic transplantation for the cure of leukemia: from its biology to clinical translation. Blood. 2016;128 (23):2616-2623.

29. Muntasell A, Ochoa MC, Cordeiro L, et al. Targeting NK-cell checkpoints for cancer immunotherapy. Curr Opin Immunol. 2017;45:73-81.

30. Carlsten M, Jaras M. Natural killer cells in myeloid malignancies: immune surveillance, NK cell dysfunction, and pharmacological opportunities to bolster the endogenous NK cells. Front Immunol. 2019;10:2357.

31. Sanchez-Correa B, Gayoso I, Bergua IM, et al. Decreased expression of DNAM-1 on NK cells from acute myeloid leukemia patients. Immunol Cell Biol. 2012; 90(1):109-115. 